\title{
An Analytical Business Process Modelling Language: An Illustrative Example
}

\author{
Azeem Lodhi \\ Fujitsu IS Lab \\ Institute for Technical and Business Information: \\ Department of Computer Science \\ University of Magdeburg, Germany \\ firstname.lastname@ovgu.de
}

\author{
Stefan Wind \\ Fujitsu IS Lab \\ Magdeburg Research and Competence Cluster: \\ Department of Computer Science \\ University of Magdeburg, Germany \\ firstname.lastname@ts.fujitsu.com
}

\author{
Veit Köppen \\ Institute for Technical and Business Information: \\ Department of Computer Science \\ University of Magdeburg, Germany \\ firstname.lastname@ovgu.de
}

\author{
Gunter Saake \\ Institute for Technical and Business Information: \\ Department of Computer Science \\ University of Magdeburg, Germany \\ firstname.lastname@ovgu.de
}

\begin{abstract}
Fierce competition exists between companies due to globalization where even small companies have a reach to wider international markets using the internet (e-business). Evaluation of business processes is very important for analysis and improvement for an organization. Different methods are used to evaluate the performances of business processes. However, there is insufficient support to evaluate the processes with detailed graphical representations. In this paper, we focus on the relation of evaluation of business processes with its representation. We follow the design science methodology in order to provide business process models for performance analysis of processes. We also provide the constructs and patterns of business process modelling for analysis and improvement of business processes. The analytical business process modelling language is highlighted with the help of a case study.
\end{abstract}

Keywords-business process, modeling, BPMN, evaluation, analysis, improvement, management

\section{INTRODUCTION}

Fierce competition exists between companies due to globalization where even small companies have a reach to wider international markets using the internet (e-business). Consider the example of purchasing a product by a consumer. He has many options like purchasing from the different retail stores, or over the internet from big brand companies to small manufacturers. This has not only increased the competition between companies, but also raised the expectation of the customers. Therefore, enterprises seek new ways to provide innovative and quality services to satisfy the customer's needs.

The changes in the business environment have also reflections in enterprises, where enterprise executives try to streamline (improve) their organizational structure and operations. This is carried out through effective design and analysis of operations. This requires understanding of operations and their performance evaluation.

Decision makers are interested in more details of processes for performance analysis. These details are necessary in order to identify deficiencies and improve the processes. Therefore, a class of users (like managers and supervisors) requires descriptive business process models.

We surveyed the existing solutions, modelling languages, and methods. However, these modelling languages do not provide support or evaluate performance of processes. Most of the modelling languages are devised for the development of information systems [1], as indicated in scientific literature and surveys [13]. Usage of same (abstract representations) methods like charts and graphs are not adequate/suitable to identify deficiencies and make decisions about process elements. Therefore, new detailed graphical models are required for this purpose.

We see the scope of an innovative artefact to solve the problems related with business process analysis and improvement. Therefore, our objective is to provide a new representation of business processes for performance analysis and to help managers in making decisions. In [11], we identified the characteristics of analytical business process modelling language for analysis and improvement.

We followed the design science research methodology [15] and the guidelines of design science as discussed in [7]. In this paper, we discuss different aspects for the specification of our proposed modelling language and provide an illustrative example of the analytical modelling language.

The structure of this paper as follows: we discuss the business context and evaluation of business processes in Section II. Visualization and representation of processes are discussed in Section III, where we also discuss the need of a modelling language for performance analysis. In this paper, we extend our earlier work [11,14] with more formal specification of analytical business process modelling language. We discuss our proposed modelling language in Section IV and explain it with the help of a case study in Section $\mathrm{V}$ using business process modelling notations (BPMN). In Section VI, we discuss the related work in this field followed by Section VII, which summarizes our paper and provides an outlook as well. 


\section{BUSINESS PROCESS \& EVALUATION}

Enterprise goals and objectives are achieved by carrying out business operations in a specific way. This specific way is called business process which can be viewed abstractly as fulfilling customer demands. Business processes are the most important elements of enterprises because it is the design of processes which have a significant impact on the overall quality of a product and success of the enterprise. Different methodologies are devised to address different aspects of business processes (from management to improvement such as business process reengineering and business process improvement [25]). Some definitions of business processes and their elements are discussed in a meta-model in [13]. However, here, we take the definition of Harrington from the business process improvement perspective [25], as follows.

"A business process consists of a group of logically related tasks that use the resources of the organization to provide defined results in support of the organization's objectives."

In this definition, the resources of organization and related tasks are key elements to fulfil the organization's objectives. The effective utilization of resources and structure of tasks are important for improvement. In [14], we have discussed different phases of business process lifecycle from evaluation and improvement perspective. Enterprises want to improve their business processes. However, before the improvement, evaluation of business process is very important and carried out at the first.

Evaluation is an important element in businesses as it is used as a tool to control and improve the processes. Different methods are used for this purpose which ranges from economics, statistics fields to computer science. In computer science focus is to provide support in carrying out business operations (automation), storage (databases), computations (mining methods), and their corresponding representations for communication (graphical models). Here, we focus on evaluation of businesses which involve computation and their corresponding representation.

Different stakeholders are involved in enterprises at different levels. These stakeholders evaluate the processes of an enterprise at different levels. In [12], stakeholders, levels, and their participation in processes with their requirements are explained in detail. Executives are interested in an abstract level evaluation like overall profit and losses. These abstract evaluations are accompanied with textual descriptions and graphical charts (like statistical charts, reports). Different trends and projections are also made (estimated) for the future. Managers evaluate processes at lower level with more details about activities and resources. However, at lower levels, the same statistical charts are used, but with different details (just focusing on certain processes or units). At this level, we need process models with more information regarding the performance of employees and structure of activities with respect to performance issues.

The performance is usually evaluated in the form of quantitative measurements and qualitative indicators. In evaluation, dimensions play an important role. This can be imagined if we do not consider a particular dimension in the design process which would be important for customer such as time dimension, cost dimension, or quality. In order to achieve the real benefits of evaluation all important dimensions and their attributes should be part of the evaluation. We should consider different performance characteristics in evaluating a product, process, or employee. Therefore, here we provide different dimensions and their characteristics which are common in practice.

TABLE I. DIMENSIONS AND CHARACTERISTICS

\begin{tabular}{|c|c|c|}
\hline Dimensions & Characteristics & Related elements \\
\hline Time & $\begin{array}{c}\text { Idle time, processing } \\
\text { time, operating time, } \\
\text { and completion time }\end{array}$ & $\begin{array}{c}\text { Activities, resources } \\
\text { (human and } \\
\text { organizational) }\end{array}$ \\
\hline Cost & $\begin{array}{c}\text { Operating cost, } \\
\text { material cost, and labor } \\
\text { cost }\end{array}$ & $\begin{array}{c}\text { Activities, resources } \\
\text { (human and } \\
\text { organizational) }\end{array}$ \\
\hline Quality & $\begin{array}{c}\text { Complaints, iterations, } \\
\text { and number of } \\
\text { customers }\end{array}$ & $\begin{array}{c}\text { Activities, resources, and } \\
\text { products }\end{array}$ \\
\hline
\end{tabular}

In evaluation, it is important for companies to have an end-to-end picture of processes, like from an abstract level to lower level details. This is important to evaluate the overall impact of changes in processes. Depending on the granularity level and stakeholder requirements, different dimensions and their characteristics become important for analysis. This makes the inclusion and filtration of particular data from the evaluation (and their perspectives). Based on the perspective requirements, other dimensions can be further included or divided into the hierarchies (like time in seconds, minutes, and hours) or into further classes based on threshold (like high cost and low cost).

\section{VISUALIZATION}

The communication of concepts related to business processes among stakeholders is important. Different ways are used for this purpose like textual descriptions and graphical techniques. Graphical techniques are used to visualize concepts/data for communication and analysis. Being graphical in nature, they provide an intuitive understanding about concepts/data. Visualization methods are used as an effective communication tool and help stakeholders to make decisions.

In [16], visualization is defined as "a cognitive activity, facilitated by external visual representations from which people build an internal mental representation of the world". Some authors also include the cognitive process and the process of building graphical models in visualization definition [16]. External visual representation can be charts or graphical models built with the help of information technology. Whereas, world means the data, information, and concepts. In [4], the author has differentiated between different visualization types like data, scientific, and information. An example of information visualization is represented in Figure 1 from [16]. 


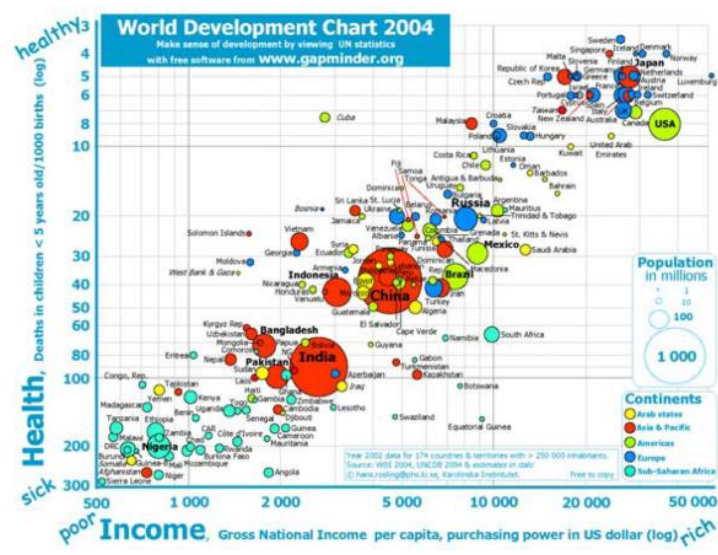

Figure 1. Information visualization from [16]

We can define visualization as a process of building graphical representations of concepts. Such representations help the stakeholder for better understanding and communication.

Different visualization techniques are also employed to evaluate businesses. These techniques depend on the user requirement which includes the goal of evaluation, level of detail, and dimension. The goal of the evaluation means what we want to measure. Depending on the goal of the evaluation, the data is prepared for visualization and different views are built. This data can be operational/raw data as in the case of data visualization or abstract as information visualization.

Different graphical models are built in order to manage business operations in a company. The field which addresses the issues related to management of business operations and graphical models is called business process modelling. Business process modelling helps in communication of business operations with stakeholders from the identification/design of processes to their automation, and then afterwards their further analysis and improvement. Graphical models built in different phases of business process management are discussed in [13].

At an abstract level, different statistical graphs (diagrams) are used to visualize the trends such as histograms (bar charts, line charts etc.). Similarly, same diagrams are used at lower levels with different details as well. The process managerial level is an important level as the changes carried out at lower levels are reflected as results at upper levels. This level requires more details in business process models. In order to solve this problem, we propose an analytical business process modelling language.

\section{Analytical Business Process Modeling LANGUAGE (ABPML)}

We design analytical business process modelling language (ABPML) by performing a thorough analysis of business process performance analysis requirements [11], existing modelling languages [13], and different analytical tools (like ProM [22], EVS [8]). We do not only include the best of breed features, but also include some innovative features which will provide help in analysing business processes and identifying deficiencies. Therefore, problem/solution search space is well investigated and considered in designing the new modelling language.

In design science, a language specification includes constructs, models, method, and instantiation components. Therefore, we address these components for specification of our proposed analytical modelling language. These are correspondingly discussed in the following.

Construct provides the vocabulary and symbols which are used to represent a problem or a solution [7] or just representing a situation. Constructs are used to represent the tangible or intangible elements of a process. They are used to represent activities, process participants (resources, places), events (communication between elements), gateways (decisions), and other involved objects (materials, orders). Therefore, they are basic building blocks of a graphical model. Semantic defines the meaning of symbols and relationship with each other.

We use the basic constructs of Business Process Modelling Notations (BPMN) as described in BPMN standard [2]. The research rigour for BPMN is discussed in Section V. However, for analytical modelling language, we propose some other constructs as an extension and also define the context in which some of basic constructs have different semantics (meanings and relations). Those constructs are used only in a defined context for performance analysis.

To accommodate different requirements, we combine constructs to build models for analysis in different perspectives, and called them as patterns. Different allowed combinations are explained here which also define the method of constructing the models in the analytical process modelling language. Depending on the user's requirements, models are built at different level of granularity to facilitate the understanding of processes. We further explain these constructs and their semantics with the help of our proposed patterns.

\section{A. Modelling Patterns}

Patterns are used to share knowledge and solve the problems [27]. Therefore, in our context, we define patterns as a combination of constructs to analyse the process and its elements in a particular perspective for improvement.

Each pattern intends to analyse performance with a particular focus involving certain dimensions and their attributes. Here, we provide four patterns for visualization of business process performance (with respect to business process modelling language). We consider these four patterns as the most important and frequent in business process analysis.

The executional data (or process trace data $[12,26]$ ) can be used to specify the usage of which activities, organizational resources, and involved elements add more value to an enterprise. Enterprises can define their own metrics. Based on this information, activities, organization 
resources, and involved elements are classified in a particular dimension and correspondingly represented using patterns.

1) Time Pattern: Time pattern focuses on representation of process element's performance from time aspect. A few classes and characteristics in this dimension are classified in Section II and represented in the next Section V with BPMN. Some examples are idle time and working time. Gantt charts like representations are easier for understanding of stakeholders; however other representations can also be used.

2) Cost Pattern: Cost is an important factor in business processes. This pattern observes the performance of process elements from cost and other related aspects involving material and other resources. Different colours can be used to distinguish between high cost and low cost elements of process. Similarly, process elements can also be grouped based on the cost incurred by them.

3) Path Pattern (Time-Cost Pattern): The time-cost pattern is helpful to decide at which path the execution will be successful. In case of different available paths, a path with company best practice can be coloured to distinguish it from other paths (options).

4) History Pattern: History path pattern represents which path is taken in most of the executions. A thick edge represents that this particular path is taken by most of the cases during execution compared to a thin edge path which represent the opposite.

The classification of process elements based on performance depends on the metrics used in the enterprise. Overall average values can be used for this classification and threshold values can be set. We recommend that only few classes should be defined for less cognitive loads of models.

Based on this information, different colors can be used to indicate the effect of the business objects like green for optimal cost, yellow for high cost, and red for very high cost. Similarly, these classes can be represented in other dimensions as well like quality and time. Although the relation of cost and time, is not as simple as discussed in [24].

Our current pattern catalogue is not meant to be complete, as different pattern can be created based on requirement and creativity of a user/analysis. A pattern catalogue can be made for enterprise performance evaluation and analysis. Due to space limitation, we do not include some of our other proposed patterns here.

\section{AN ILLUSTRATIVE EXAMPLE USING BPMN}

Instantiation demonstrate the feasibility of proposed modelling language to solve the problem and its benefit. For this purpose, we take an example of a small case study.

We have chosen business process modelling notations (BPMN) for demonstration of proposed analytical modelling language. This is due to the reason that BPMN is rigorously defined and has widely accepted as a standard (defined by OMG) for modelling/communicating business processes. It is implemented in different modelling tools and rich in representation. Similarly, various extensions are also proposed in order to suit different business needs.

BPMN graphical notations are divided into four basic categories [2]. These categories are discussed briefly as follow.

Flow objects consist of activities, involved decision nodes for their order (sequential, parallel, iterations), and events of processes. Connecting objects as the name implies are used to connect the activities and other elements with each other using different arrows which represent messages and associations between them. This core set of elements define the control flow perspective of processes.

Different modeling elements are grouped through Swimlanes which use pools and lanes [2]. A Pool is used to represent process participants while lanes are used to partition these participants and their activities from one to another. A process participant can either be organizational entities within an organization or different organizations for collaboration in a process. Mostly, organizational perspective is provided by using Swimlanes constructs. In BPMN, additional information about the process such as involved data object and guidelines for operations are provided by artifacts. These elements consist of data objects, annotations, and group constructs.

\section{A. Case Study}

Consider an online purchasing scenario where a customer arrives on a web portal. A customer selects a particular product to purchase. In our example, it is a customized personal computer (desktop, two display devices, and accessories). He adds the products into a shopping basket and fulfills the order request form with shipping details. Then, he processes the online payment form. Several other processes are involved in processing this scenario like fulfilling an order request, transfer of a payment, manufacturing the product, and shipping process.

Once the order form is completed, it is sent to the manufacturer/retailer for further processing. On the manufacturer/retailer side, the required product is checked in the stock. If the required products is not in stock, then a production order is prepared for manufacturing the products (assume that ready products are not available in our case).

In manufacturing product scenario, first the raw material is collected from the store. Afterwards, it is manufactured. Quality inspectors examine the quality of the manufactured product. This manufacturing process is abstractly shown in Figure 2.

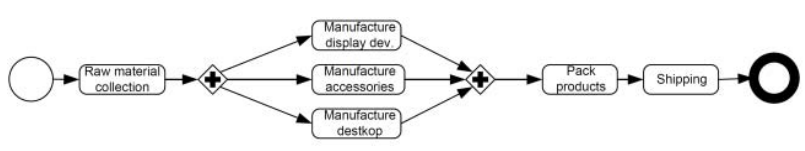

Figure 2. Abstract manufacturing process

Assume display devices are manufactured in time and ready for shipping them to customer whereas the computer desktop takes four weeks for manufacturing. Similarly, accessories take only one week. Once they are ready, they 
are shipped together to the customer. Therefore, the overall order is completed and marked as completed. When all these steps are recorded in information systems, we can use this data to analyze the performance of organizational elements, ordering of activities and other involved objects.

The whole process can be analyzed with abstract details like how much time it took to complete the order as shown in the Figure 3. However, at managerial level the process can be analyzed with more detailed representation that will help to identify the deficiencies and improve it. For this detailed representation, here we will discuss our proposed patterns in the context of this example.

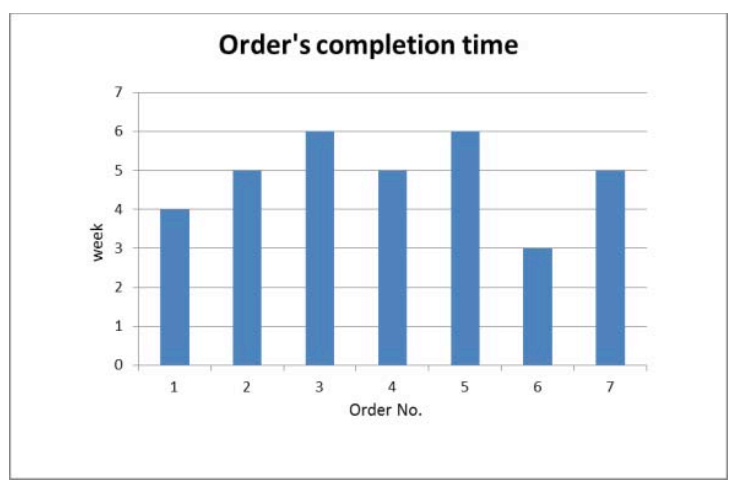

Figure 3. Abstract details about order completion

In BPMN, Swimlanes (pool and lane) are used to represent process participants and their interaction during execution. We propose to use Swimlanes not only to see participant interaction, but also the performance of organizational resources and activities. Based on collected data, process participant performance should be computed and their lanes should be colored (like green, yellow, and red). Similarly, activities can also be aligned using Swimlanes based on their computation in a particular dimension and their attributes. Consider Figure 4, where three classes are defined in cost dimension to arrange the activities of processes and their involved elements.

Similarly, different dimension attributes can also be defined as pools where lanes represent further classes of these attributes as shown in Figure 5. In Figure 5, activity manufacture display dev. is represented in two different attributes of time dimension. This figure shows that the actual operating time would be very short, but with high idle time. For simplicity, we have not shown different other involved activities in Figure 5. Moreover, different dimensions and their attributes can be combined with one another for further business process analysis.

When we represent performance details using Swimlanes in the BPMN model, we can find out which activities are consuming time and taking high costs. Afterwards, these activities can be further investigated to identify their deficiencies for improvement.

Similarly, the path and history patterns can also be represented by coloring the edges (representing best practice) and changing the thickness of connecting objects (edges).

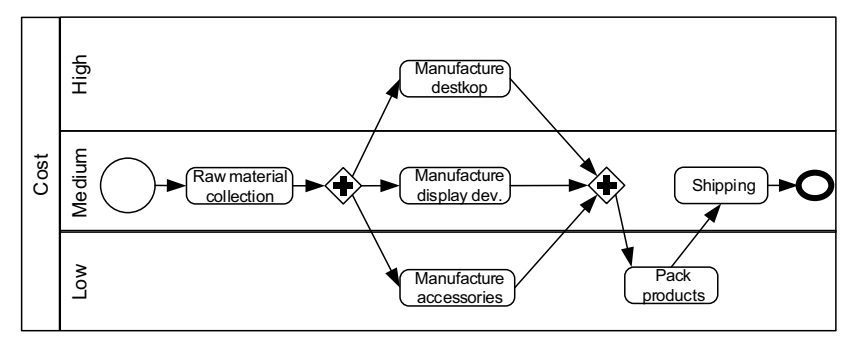

Figure 4. cost based representation of processes
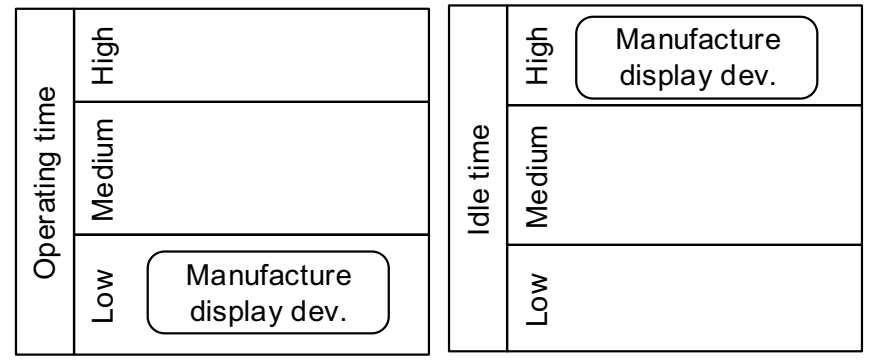

Figure 5. cost based representation of processes

Based on these extended notations, we represent an extended business process model of our example in Figure 6. The colors red, yellow, and green represent the activities with high, medium, and low cost respectively. In order to avoid confusion, in this figure, we only coloured the activities to represent the performance aspects whereas Swimlanes are kept with their classical BPMN semantics [2] such as process participants.

\section{RELATED WORK}

Our work is compared and contrasted with existing modelling languages and methods. However, most of the research in business process modeling domain is related to the information systems, like development [9] or improving the IT services with respect to business processes [20]. There is limited research with the focus on performance analysis using business process models. Some approaches analyze business processes after execution, however, in those approaches same models are used which are conceptualized for information system development like some process mining [21] tools use Petri nets [19]. Therefore, the solution provided them are only on ad-hoc basis which are not appropriate. An evaluation of different modelling languages and available tools are provided in [13].

Different approaches are categorized in formal and semiformal approaches for analysis and optimization in a survey [23]. Their survey shows the lack of business process modeling languages for post executional phases. However, they do not provide any extensions or examples of modeling languages which we have provided in this paper. The concept of excluding activities at the abstract level and including them at the detailed level is common in most of the business process modeling languages, however in $[2,6]$ it is 
represented for detailed understanding of processes whereas in [3], it is discussed at the attribute level. Different views of models are generated based on the environment (role) of execution as discussed in [5], however, they are discussed from the software process perspective, irrespective to business processes.

An interesting work in BPMN domain was presented in [17] where an approach to transform business process dimensions (time, business rules, and information) into BPMN constructs was discussed. However, author focus was not on the performance analysis of business processes. Here, we provide different patterns for better understanding and representation of business process for performance evaluation. In [14], we have extended the BPMN meta model for performance evaluation of business processes after execution. Similarly, there are also some other attempts to extend BPMN models in different dimension like knowledge dimension [18] and modeling process goals and its measures in $[10]$.

Different business process management suites also provide the cockpits to represent the performance metrics in graphical forms like histograms, radial graphs, and several other techniques. However, these representations are abstract representations without providing information about structure. Similarly, process mining tools (like ProM [22], EVS [8]) also exhibit performance metrics through different graphical models. These approaches lack the support of business process modeling language to provide process perspective for improvement.

\section{SUMMARY AND OUTLOOK}

In this paper, we presented a design science artefact (a modelling language) to solve the problem of performance analysis of business process. We followed the design science guidelines for specification of modelling language. We presented four patterns of our proposed modelling language for performance analysis of business processes. These patterns are explained with the help of an example using a rigorously defined modelling language (BPMN). We extended BPMN for performance analysis.

In the future, we plan to add further patterns in our pattern catalogue for analysis and improvement. We will also do the quantitative and qualitative evaluation of proposed modelling language. A tool will also be developed for generating and presenting the proposed modelling language for analysis and improvement purpose.

\section{REFERENCES}

[1] G. Booch, J. Rumbaugh, and I. Jacobson, Unified Modeling Language User Guide, 2nd ed. Addison-Wesley, May 2005.

[2] BPMI.org and OMG, "Business Process Modeling Notation Specification, Final Adopted Specification," Object Management Group, OMG Headquarters, 140 Kendrick Street, Building A, Suite 300, Needham, MA 02494, USA, February 2006. [Online]. Available: http://bpmn.org/Documents/OMG_Final_Adopted_BPMN_1-0_Spec 06-02-01.pdf

[3] T. R. Browning, "The many views of a process: Toward a process architecture framework for product development processes," System
Engineering, volume. 12, No. 1, pp. 69-90, 2009.

[4] E. H. Chi, "A Taxonomy of Visualization Techniques Using the Data State Reference Model," in Proceedings of the IEEE Symposium on Information Vizualization 2000 (INFOVIS '00). Washington, DC, USA: IEEE Computer Society, 2000, pp. 69+. [Online]. Available: http://portal.acm.org/citation.cfm?id=857691

[5] D. Correal and R. Casallas, "Using domain specific languages for software process modeling," in ACM OOPSLA, Workshop on Domain-Specific Modeling, 2007. [Online] Available: http://citeseerx.ist.psu.edu/viewdoc/summary?doi=10.1.1.102.64

[6] A. Dreiling, M. Rosemann, W. van der Aalst, and W. Sadiq, "From conceptual process models to running systems: A holistic approach for the configuration of enterprise system processes," Decision Support Systems, vol. 45, no. 2, pp. 189-207, 2008. [Online] Available: http://www.sciencedirect.com/science/article/B6V8S4N43RHR-1/2/fbc3c60644d31ffffb4665412e09fe5e

[7] A. R. Hevner, S. T. March, J. Park, and S. Ram, "Design science in information systems research," MIS Q., vol. 28, no. 1, pp. 75-105, Mar. 2004.2 [Online]. Available: http://dl.acm.org/citation.cfm?id=2017212.2017217

[8] J. E. Ingvaldsen, J. A. Gulla, O. A. Hegle, and A. Prange, "Empirical business models," in CAiSE Short Paper Proceedings, ser. CEUR Workshop Proceedings, vol. 161. CEURWS, 2005.

[9] S. Klink, Y. Li, and A. Oberweis, "INCOME2010 - A toolset for developing process-oriented information systems based on Petri nets," in Simutools '08: Proceedings of the $1^{\text {st }}$ international conference on Simulation tools and techniques for communications, networks and systems \& workshops. Belgium: ICST, 2008, pp. 1-8.

[10] B. Korherr and B. List, "Extending the EPC and the BPMN with business process goals and performance measures," in Proceedings of the 9th International Conference on Enterprise Information Systems (ICEIS)(3), J. Cardoso, J. Cordeiro, and J. Filipe, Eds., 2007, pp. 287-294.

[11] A. Lodhi, V. Köppen, and G. Saake, "Post execution analysis of business processes: Taxonomy and challenges," University of $\begin{array}{llll}\text { Magdeburg, } & \text { Tech. } & \text { Rep. } & \text { 9, }\end{array}$

[12] A. Lodhi, V. Köppen, and G. Saake, "Business process improvement framework and representational support," in Proceedings of The Third International Conference on Intelligent Human Computer Interaction (IHCI), ser. Advances in Intelligent and Soft Computing. Prague, Czech Republic: Springer, August 2011.

[13] A. Lodhi, V. Köppen, and G. Saake, "Business process modeling: Active research areas and challenges," University of Magdeburg, Tech. Rep. 1, 2011.

[14] A. Lodhi, V. Köppen, and G. Saake, "An extension of bpmn metamodel for evaluation of business processes," J. Riga Technical University, vol. 43, pp. 27-34, 2011.

[15] S. T. March and G. F. Smith, "Design and natural science research on information technology," Decis. Support Syst., vol. 15, no. 4, pp. 251-266, Dec. 1995. [Online]. Available: http://dx.doi.org/10.1016/0167-9236(94)00041-2

[16] R. Mazza, Introduction to Information Visualization, 1st ed. Springer, 2009. 
[17] L. Penicina, "The approach of transformation between business process dimensions in BPMN modeling tool," in Proceedings of the 15th International Conference on Information and Software Technologies, Lithuania, Kaunas, April 2009, pp. 72-81.

[18] I. Supulniece, L. Businska, and M. Kirikova, "Towards extending BPMN with the knowledge dimension," in Enterprise, BusinessProcess and Information Systems Modeling, ser. Lecture Notes in Business Information Processing, W. van der Aalst et.al., Ed. Springer, 2010, vol. 50, pp. 69-81. [Online]. Available: http://dx.doi.org/10.1007/978-3-642-13051-9

[19] W. van der Aalst, "The application of Petri nets to workflow management." The Journal of Circuits, Systems and Computers, vol. 8, pp. 21-66, 1998 .

[20] W. van der Aalst, "Business alignment: Using process mining as a tool for delta analysis and conformance testing," Requirement Engineering, vol. 10, no. 3, pp. 198-211, 2005. [Online]. Available: http://portal.acm.org/citation.cfm?id=1342177

[21] W. van der Aalst, H. A. Reijers, A. Weijters, B. van Dongen, A Medeiros, M. Song, and H. Verbeek, "Business process mining: An industrial application," Information Systems, vol. 32, no. 5, pp. 713$732,2007$.

[22] W. van der Aalst, B. van Dongen, C. Güunther, A. Rozinat, H. Verbeek, and A. Weijters, "ProM: The process mining toolkit," in Proceedings of the BPM 2009 Demonstration Track, ser. CEUR Workshop Proceedings, A. K. A. de Medeiros and B. Weber, Eds., vol. 489. CEURWS. org, September 2009, pp. 1-4. [Online]. Available:

http://ceur-ws.org/Vol-489/paper3.pdf

[23] K. Vergidis, A. Tiwari, and B. Majeed, "Business process analysis and optimization: Beyond reengineering," Systems, Man, and Cybernetics, Part C: Applications and Reviews, IEEE Transactions on, vol. 38 , no. 1 , pp. 69-82, 2008.

[24] M. J. Vullers, P. Kleingeld, M. Loosschilder, and H. A. Reijers, "Performance measures to evaluate the impact of best practices." in Proceedings of Workshops and Doctoral Consortium of the 19th International Conference on Advanced Information Systems Engineering (BPMDS). Tapir Academic Press Trondheim, 2007, pp 359-368.

[25] J. H. Harrington, Business Process Improvement: The Breakthrough Strategy for Total Quality, Productivity, and Competitiveness McGraw-Hill, April 1991

[26] A. Khan, A. Lodhi, V. Köppen, G. Kassem, and G. Saake. Applying Process Mining in SOA Environments. In Asit Dan, Frédéric Gittler, and Farouk Toumani, editors, Service-Oriented Computing (ICSOC/ServiceWave) 2009 Workshops, volume 6275 of LNCS, pages 293-302. Springer, November 2009.

[27] A. Khan, C. Kästner, V. Köppen, and G. Saake. Service Variability Patterns in SOC. Technical Report 5, University of Magdeburg, Germany, May 2011.

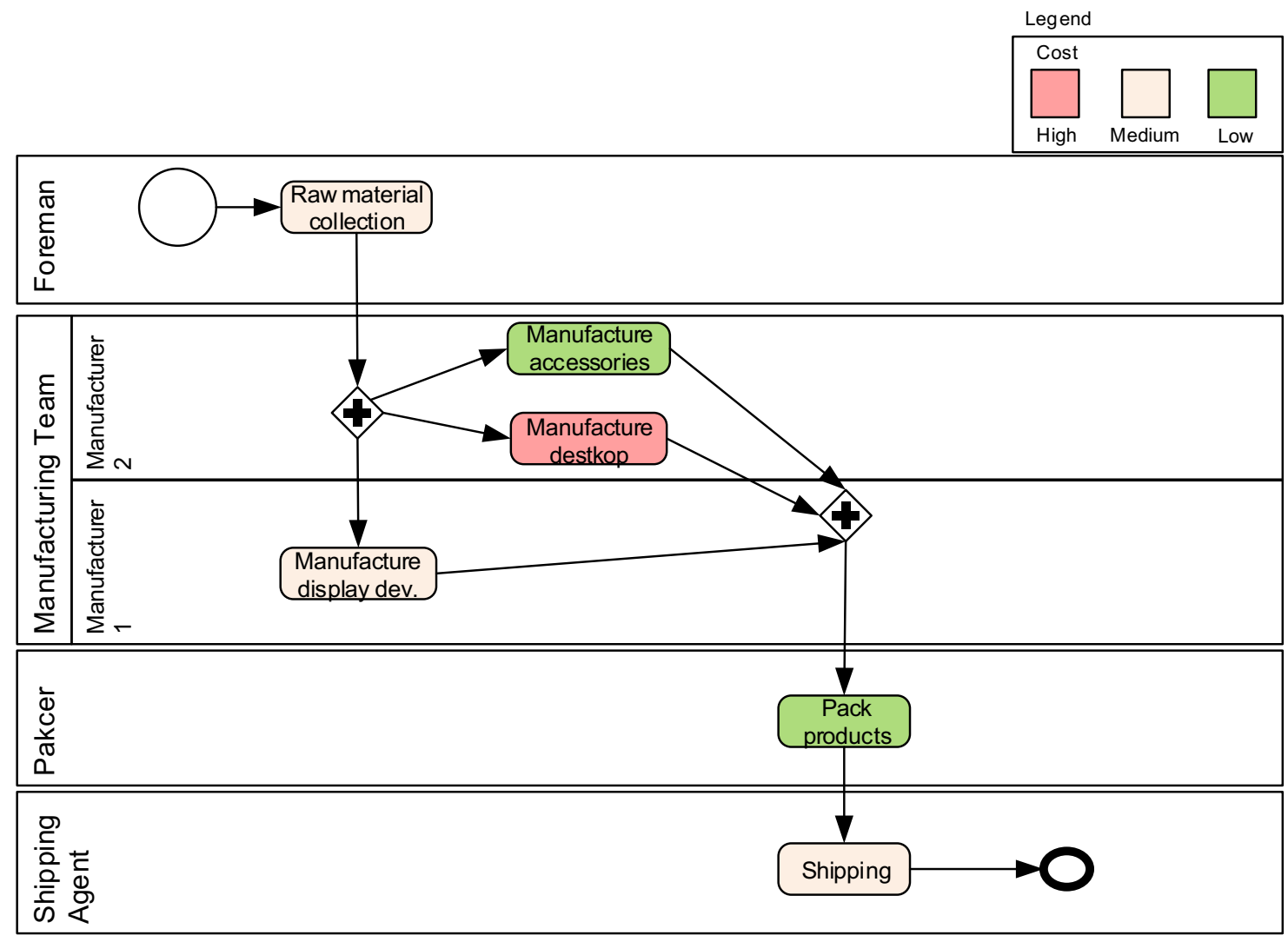

Figure 6. Extended representation of example using BPMN 